\title{
Impacto e aderência de indivíduos com diabetes no Programa Academia da Cidade
}

\section{Impact and adherence of individuals with diabetes in the Academia da Cidade Program}

\section{AUTORES \\ Débora Cristina Jubilini ${ }^{1}$ (D) \\ Adriana Bosco $^{1}$ (D) \\ 1 Santa Casa de Misericórdia de Belo Horizonte, Instituto de Ensino e Pesquisa, Belo Horizonte, \\ Minas Gerais, Brasil. \\ CONTATO \\ Débora Cristina Jubilini \\ deborajubilini@gmail.com \\ Rua Salvador Gurgel, n. 79 / 101, Santa Cruz, \\ Belo Horizonte, Minas Gerais, Brasil. \\ CEP: $31155-180$. \\ DOI}

$10.12820 /$ rbafs. $25 \mathrm{e} 0136$

\section{(cc) BY}

Este trabalho está licenciado com uma Licença Creative Commons - Atribuição 4.0 Internacional.

\begin{abstract}
RESUMO
Uma epidemia de Diabetes Mellitus tipo 2 (DM2) está em curso e uma grande proporção de pessoas com essa doença não pratica exercícios físicos regularmente. Em Belo Horizonte, o Programa Academia da Cidade (PAC) se apresenta como uma estratégia de melhoria da qualidade de vida da população, através da prática de exercícios físicos. Logo, é relevante compreendermos o impacto deste na saúde de indivíduos com e sem diagnóstico de DM, ao longo do tempo. Para análise da aderência foram utilizadas as chamadas de frequência e para as medidas antropométricas utilizou-se as Planilhas de Avaliação Física do Programa. Foram avaliados 812 usuários, divididos nos grupos: "diabéticos" (GD) e "nāo-diabéticos" (GND). A avaliação física foi feita no início, 6 e 12 meses após inserção às atividades. A presença às aulas foi semelhante entre os grupos GD e GND. No momento de entrada, as medidas de pressão arterial sistólica (PAS), peso (P), circunferência abdominal (CA) e índice de massa corporal (IMC) do GD foram significativamente maiores do que GND ( $p=0,008$, $0,001,<0,001$ e $<0,000$, respectivamente). Após 12 meses, GD e GND apresentaram diminuição significante de CA ( $p=0,037$ e $p=0,005$, respectivamente). Em conclusão, no PAC da Regional Nordeste de Belo Horizonte, após 12 meses, GD e GND apresentaram aderência semelhante às aulas, havendo redução significativa para CA. Sugere-se um estudo por maior tempo para identificar resultados mais robustos às demais variáveis.
\end{abstract}

Palavras-chave: Diabetes mellitus; Exercício físico; Atenção primária à saúde.

\begin{abstract}
An epidemic of Type 2 Diabetes Mellitus (DM) is ongoing and a large proportion of people diagnosed with the disorder do not exercise regularly. In Belo Horizonte, the Academia da Cidade Program is a strategy to improve the quality of life of the population through the practice of physical exercises. Therefore, it is important to understand the impact of the program on the health of individuals with and without a diagnosis of DM over time. For the analysis of adherence, we used attendance lists, and, for the anthropometric measures, we used a physical evaluation protocol from the Program. We assessed 812 members of the Program, classified into two groups: "diabetic" (DG) and "non-diabetic" (NDG). We applied the physical examination at the baseline, 6 and 12 months after the beginning of the exercise practice. Class attendance was similar between the DG and NDG groups. At the baseline, the measurements of systolic blood pressure, body weight, waist circumference, and body mass index were significantly greater for $D G$ than for $N D G(p=0.008,0.001$, $<0.001$ and $>0.001$, respectively). After 12 months, DG and NDG showed significant waist circumference reduction in comparison to the baseline ( $p=0.037$ and $p=0.005$, respectively). In conclusion, in the Academia da Cidade Program of the Northeast Region of Belo Horizonte DG and GND showed similar attendance to classes, with a significant reduction for waist circumference after a year. However, a longer study is desirable to identify significant results for the other variables.
\end{abstract}

Keywords: Diabetes mellitus; Exercise; Primary health care.

\section{Introdução}

O DM 2 é considerado um problema de saúde pública, uma epidemia ainda em ascensão que segue em paralelo com a obesidade ${ }^{1}$. Em 2015, o Brasil foi classificado como o quarto país com maior número de pessoas com DM entre 20 e 79 anos de idade ${ }^{2}$. Além disso, de acordo com o sistema VIGITEL ${ }^{3}$ (Vigilância de Fatores de Risco e Proteção para Doenças Crônicas por Inquérito Telefônico), de 2019, em Belo Horizonte, a frequência de adultos que referem diagnóstico de DM foi de 7,5\%, sendo que tal condição aumenta com a idade e em indivíduos com menor escolaridade.

Os mecanismos que ligam o exercício físico à prevenção e ao tratamento do DM envolvem, principalmente, a redução da adiposidade corporal, a melhora da sensibilidade à insulina, além do aumento do gasto energético, da força muscular e da capacidade cardiorrespiratória ${ }^{4-6}$. Entretanto, apesar do exercício físico ser 
o elemento chave para a prevenção e administração do $\mathrm{DM}$, a maioria dos indivíduos com essa condição não praticam uma atividade física regular ${ }^{6,7}$.

Atualmente, $38,1 \%$ da população brasileira pratica pelo menos 150 minutos de atividade física na semana no tempo livre ${ }^{3}$. Nos Estados Unidos (EUA), estima-se que 39\% dos adultos com DM estejam fisicamente ativos, em comparação com 58\% que não possuem tal diagnóstico. Dentre os norte-americanos com mais de 60 anos e com DM, apenas $28 \%$ são fisicamente ativos ${ }^{1}$. De acordo com Estudo Longitudinal de Saúde do Adulto (ELSA-Brasil $)^{8}$, apenas $17,8 \%, 15,1 \%$ e $13,9 \%$ dos indivíduos que relataram dislipidemia, hipertensão arterial sistêmica (HAS) e DM, respectivamente, seguem as recomendações de atividade física (150 minutos de atividade física de intensidade moderada, ao longo da semana).

Em 2006, foi implementado em Belo Horizonte as Academias da Cidade como estratégia para propiciar a melhoria da qualidade de vida da população por meio de ações de incentivo à prática de exercícios físicos e de uma alimentação saudável ${ }^{9}$. Em 2011, as Academias da Cidade passaram a contar com o apoio do Governo Federal, que lançou o Programa Academia da Saúde com o objetivo de contribuir para promoção da saúde com a criação de infraestrutura, equipamentos e quadro de pessoal qualificado para a orientação de práticas corporais de atividade física e de lazer ${ }^{10}$.

Além de auxiliar as pessoas a diminuírem o tempo em comportamento sedentário, os Profissionais de Educação Física enfrentam um outro desafio: aumentar a aderência à prática do exercício físico. Situação constantemente ameaçada por inúmeros motivos, que vão desde a falta de tempo, passando pela ausência de motivação, agudizaçāo de problemas de saúde até dificuldade de acesso às academias ${ }^{11}$.

Com o intuito de avaliar o conhecimento e os fatores relacionados à mudança de comportamento no controle do DM, Mendes et al. ${ }^{12}$, entrevistaram 872 idosos residentes na cidade de São Paulo. Dentre os entrevistados, $17,6 \%$ se auto referiram possuir DM e desses, apenas $15,7 \%$ participavam de grupos de educação para o controle da doença. As atitudes mais referidas pelos indivíduos para controle do DM foram "tomar medicação oral de rotina" $(60,8 \%)$ e "fazer dieta alimentar" $(52,1 \%)$. A prática de atividade física regular foi a menos citada, com apenas 2,2\% dos entrevistados referindo este comportamento como parte do tratamento.

$\mathrm{O}$ PAC, em Belo Horizonte, é de frequência predominantemente feminina e acessado principalmente para melhoria da saúde, pela convivência e facilidade no acesso ${ }^{13}$. Costa et al. ${ }^{14}$, analisando o perfil clínico e nutricional dos usuários do SUS ingressantes em um PAC de Belo Horizonte, identificaram elevada prevalência de indivíduos hipertensos (41,6\%), com excesso de peso $(70,6 \%)$ e riscos metabólicos associados à obesidade (67,6\%). Além disso, 40\% apresentavam de uma a três doenças crônicas e mais de $65 \%$ utilizavam medicamentos diariamente.

Sendo assim, este estudo tem o objetivo de analisar a aderência dos usuários inscritos no PAC e as consequências dessa nas medidas antropométricas, considerando a relevância de compreendermos o impacto deste Programa na saúde de indivíduos com e sem diagnóstico de DM.

\section{Métodos}

Trata-se de um estudo observacional, descritivo e retrospectivo, com dados coletados durante o ano de 2015, em uma regional de Belo Horizonte, da qual a autora foi Referência Técnica do Núcleo Ampliado de Saúde da Família e Atenção Básica (NASF-AB). O estudo possui Carta de Anuência assinada pela Secretaria Municipal de Saúde de Belo Horizonte e aprovação nos Comitês de Ética da Santa Casa de Misericórdia de Belo Horizonte (número do parecer: 1.962.621) e Secretaria Municipal de Saúde de Belo Horizonte (número do parecer: 2.011.842).

O PAC, em Belo Horizonte, oferta a prática regular de exercícios físicos para pessoas acima de 18 anos de idade, de forma sistematizada e supervisionada pelo Profissional de Educação Física, do Centro de Saúde de referência. Para o ingresso, é necessário avaliação física e cada usuário teve a possibilidade de realizar exercícios físicos até três vezes por semana durante 60 minutos.

A prática de exercícios físicos era organizada da seguinte maneira: todo encontro possuía $30 \mathrm{minu}-$ tos de caminhada e os demais 30 minutos deveriam ser preenchidos por estímulos aeróbios ou resistidos, contanto que esses fossem intercalados ao longo da semana. Logo, o PAC, em Belo Horizonte, ao longo da semana, oferecia aulas de exercícios combinados e aulas de exercícios aeróbios.

Para além dos exercícios, os inscritos também poderiam participar de outras ações para a promoção de saúde (campanhas de vacinação, orientação para uma alimentação saudável, combate à dengue, cessação do tabagismo, cuidados no trânsito, etc) e cidadania (participação nas comissões locais de saúde). Essas aborda- 
gens não apresentavam uma metodologia única, como ocorria com a prescrição de exercícios físicos. Os Profissionais de Educação Física poderiam definir as ações a serem ofertadas a cada mês, de acordo com as necessidades do território e de seus respectivos usuários. Os polos do Programa possuem uma estrutura de funcionamento básica: sala para atendimento individual, espaço para atividades coletivas, espaço para caminhada orientada, além de materiais como aparelho de som, colchonetes, halteres, caneleiras e outros.

Para manutenção no Programa, os usuários deveriam submeter-se a avaliações físicas periódicas e manter uma frequência às aulas. Aqueles usuários que se ausentassem por 15 dias consecutivos seriam considerados infrequentes. Além disso, na necessidade de se ausentarem, a vaga no Programa seria mantida por 3 meses. Todos os dados são registrados em uma Planilha de Avaliação Física específica do Programa. A Planilha de Avaliação Física era disponibilizada através do software Excel. As primeiras 29 colunas da planilha apresentavam informações gerais do usuário, como nome completo, endereço, telefone de contato, centro de saúde de referência e data de nascimento. As colunas seguintes, por sua vez, avaliavam o risco do indivíduo de possuir algum evento cardiovascular na prática de exercício, a presença do diagnóstico de DM, o relato de dores, uso de medicamentos, além dos hábitos alimentares, comportamentos sedentários, medidas antropométricas, risco de queda e outras informações relacionadas à saúde. Sendo assim, foram incluídos neste estudo apenas os usuários com os dados corretamente registrados e lista de chamada devidamente preenchida.

Para esta pesquisa, foram analisados os dados de pressão arterial sistólica (PAS) e diastólica (PAD) em repouso $(\mathrm{mmHg})$, aferidas no braço esquerdo, com o usuário sentado, de pernas afastadas e apoiadas ao chão, após 5 minutos; peso corporal $(\mathrm{P}-\mathrm{Kg})$ e estatura $(\mathrm{E}$ - $\mathrm{m}$ ), posicionando o usuário com os pés descalços e ligeiramente afastados na balança; circunferência abdominal $(\mathrm{CA}-\mathrm{cm})$, utilizando-se fita antropométrica e referência da cicatriz umbilical e cálculo do índice de massa corporal $\left(\mathrm{IMC}-\mathrm{Kg} / \mathrm{m}^{2}\right)\left(\mathrm{P} / \mathrm{E}^{2}\right)$.

No ano de 2015, a regional Nordeste de Belo Horizonte possuía 21 Centros de Saúde e 7 Academias da Cidade. No entanto, neste estudo, foram incluídas apenas 3 que atendiam aos critérios estabelecidos. $\mathrm{O}$ total de usuários incluídos foram divididos em 2 grupos diante auto referimento em possuir ou não DM e o relato de uso ou não de medicamentos para DM: diabéticos (GD) e nāo-diabéticos (GND).

O cálculo amostral foi construído a partir dos parâmetros de nível de confiança, margem de erro e tamanho da população, sendo o $\mathrm{n}$ amostral mínimo de 71 para GD e 147 para GND. Para análise estatística, a caracterização da amostra foi feita por tabelas de frequências e pelas medidas descritivas de média, mediana, desvio-padrão e quartis. Para verificar a normalidade consideramos o teste de Shapiro Wilk em cada variável, estratificando por grupo. Apenas PAS, no grupo com DM, foi considerada com distribuição normal. Para esta, utilizamos o teste de ANOVA e o teste de Friedman para as demais variáveis, comparando dados pareados não normais. Em todos os testes foi utilizado um nível de significância de 5\%. As análises foram realizadas no software STATA versão 12.0.

Ao longo do ano, foram ofertadas 144 aulas e o percentual de aderência foi calculado a partir do registro de presença em cada aula. As medidas antropométricas foram avaliadas no início da intervenção e reavaliadas após 6 e 12 meses.

\section{Resultados}

Foram avaliados 812 usuários, sendo $724(89,2 \%)$ pertencentes ao gênero feminino e $88(10,8 \%)$ ao masculino. Do total de participantes, $435(53,8 \%)$ possuíam mais de 60 anos de idade e 329 (40,5\%) se autodeclaravam pardos. Em relação a presença de DM, 114 (14\%) pertenciam ao GD, 5 com DM1 e 698 (86\%) foram incluídos no GND (Tabela 1). A média de idade foi de $62,98 \pm 10,74$ anos no GD e 58,82 $\pm 13,11$ no GND.

A comparação das variáveis antropométricas entre GD e GND (Tabela 2) mostram, por medianas e quartis, que no momento da entrada no estudo as medidas de PAS, P, CA e IMC foram significantemente maiores no GD ( $p=0,008,0,001,<0,001$ e $<0,000$, respectivamente) e não se observou diferença na $\mathrm{PAD}$ entre os grupos $(p=0,596)$. Após 6 meses, os indivíduos do GD mantiveram PAS, CA e IMC maiores que GND, ( $p=0,025,<0,001,0,006$, respectivamente) e, após 12 meses, somente a PAS e CA permaneceram maiores ( $\mathrm{p}$ $=0,014$ e 0,010, respectivamente).

Considerando a média da frequência às aulas, os grupos apresentaram aderência semelhante ao PAC, o GD registrou 56,7\% de presença e o GND 56,2\% (Tabela 3).

Ao longo do ano, dos 114 indivíduos do GD avaliados no momento de entrada, 71 foram reavaliados após 6 meses e 44 após 12 meses. Considerando o uso de testes não paramétricos e tendo a mediana como 
Tabela 1 - Características dos usuários do PAC, da região Nordeste de Belo Horizonte, em 2015 ( $\mathrm{n}=812$ )

\begin{tabular}{lcc}
\hline Variáveis & $\begin{array}{c}\text { Quantidade de } \\
\text { usuários }(\mathrm{n})\end{array}$ & $\%$ \\
\hline Gênero & 724 & 89,2 \\
Feminino & 88 & 10,8 \\
Masculino & & \\
Faixa etária ( $\mathrm{n}=$ 809) & 10 & 1,2 \\
18-24 anos & 39 & 4,8 \\
$25-39$ anos & 325 & 40,2 \\
40-59 anos & 435 & 53,8 \\
60 anos ou mais & & \\
Raça (n = 808) & 329 & 40,5 \\
Pardo & 233 & 28,7 \\
Branco & 226 & 27,8 \\
Negro & 5 & 0,6 \\
Amarela & 15 & 1,8 \\
Indígena & & \\
Presença de diabetes & 114 & 14,0 \\
GD & 5 & 0,6 \\
GD DM1 & 109 & 13,4 \\
DG DM2 & 698 & 86,0 \\
GND & & \\
\hline
\end{tabular}

$\mathrm{n}$ = número de usuários; $\mathrm{GD}=$ diabéticos; $\mathrm{GND}=$ não diabéticos;

DM1 = diabetes tipo 1; DM2 = diabetes tipo 2. parâmetro, observamos que, nos 6 meses iniciais, $P$, CA e IMC tenderam a uma redução, mas não foi significativa. Ao longo do ano, dos 114 indivíduos do GD avaliados no momento de entrada, 71 foram reavaliados após 6 meses e 44 após 12 meses. Considerando o uso de testes não paramétricos e tendo a mediana como parâmetro, observamos que, nos 6 meses iniciais, P, CA e IMC tenderam a uma redução, mas não foi significativa. Ao longo de 12 meses de participação no $\mathrm{PAC}$, indivíduos com DM não apresentaram diminuição estatisticamente diferente de P e IMC ( $\mathrm{p}=0,466$ e 0,168 , respectivamente) entretanto, a diminuição da CA foi estatisticamente significante $(p=0,037)$. Os valores de PAS $(\mathrm{p}=0,836)$ e $\operatorname{PAD}(\mathrm{p}=0,530)$ não apresentaram alterações significativas (Tabela 4).

Dos 698 indivíduos no GDN avaliados no momento de entrada, 453 foram reavaliados em 6 meses e 324 reavaliados após 12 meses. Assim, considerando a mediana como melhor parâmetro, após 6 meses de participação no PAC, valores de $\mathrm{P}, \mathrm{CA}$ e IMC não apresentaram uma redução estatisticamente diferente, quando comparados ao momento inicial. Após 12 meses, P e IMC ( $p=0,877$ e e 0,591, respectivamente)

Tabela 2 - Comparação das características antropométricas entre GD e GND na entrada ao PAC da região Nordeste de Belo Horizonte, em $2015(n=812)$.

\begin{tabular}{|c|c|c|c|c|c|c|}
\hline \multirow{2}{*}{ Variáveis } & Entrada $(\mathrm{t} 0)$ & $\mathrm{p}$ & 6 meses $(\mathrm{t} 1)$ & $\mathrm{p}$ & 12 meses $(\mathrm{t} 2)$ & $\mathrm{p}$ \\
\hline & $(\mathrm{n}=812)$ & $(\mathrm{t} 0)$ & $(\mathrm{n}=535)$ & $(\mathrm{t} 1)$ & $(\mathrm{n}=378)$ & $(\mathrm{t} 2)$ \\
\hline \multicolumn{7}{|c|}{ PAS (mmHg) } \\
\hline GD & $130,0(120,0-140,0)$ & \multirow[b]{2}{*}{$0,008^{*}$} & $130,0(120,0-140,0)$ & \multirow{2}{*}{$0,025^{*}$} & $130,0(122,0-140,0)$ & \multirow{2}{*}{$0,014^{*}$} \\
\hline GND & $121,0(110,0-138,0)$ & & $122,0(110,0-136,0)$ & & $122,0(110,0-136,0)$ & \\
\hline \multicolumn{7}{|c|}{$\mathrm{PAD}(\mathrm{mmHg})$} \\
\hline GD & $80,0(70,0-84,5)$ & \multirow{2}{*}{0,596} & $80,0(70,0-86,0)$ & \multirow{2}{*}{0,776} & $80,0(70,0-86,0)$ & \multirow{2}{*}{0,717} \\
\hline GND & $80,0(70,0-84,0)$ & & $80,0(70,0-84,0)$ & & $80,0(70,0-84,0)$ & \\
\hline \multicolumn{7}{|c|}{ Peso Corporal (Kg) } \\
\hline GD & $72,6(65-84,2)$ & \multirow{2}{*}{$0,001^{*}$} & $69,8(61,6-78,4)$ & \multirow{2}{*}{0,084} & $70,2(61,8-75,0)$ & \multirow{2}{*}{0,269} \\
\hline GND & $68,2(59,5-77,6)$ & & $67,3(59,5-76,2)$ & & $66,7(59-74,9)$ & \\
\hline \multicolumn{7}{|l|}{$\mathrm{CA}(\mathrm{cm})$} \\
\hline \multicolumn{7}{|l|}{ GD } \\
\hline Mulheres & $101,7(90,5-110,0)$ & \multirow{5}{*}{$<0,001^{*}$} & $100,0(90,0-106,0)$ & \multirow{5}{*}{$<0,001^{*}$} & $99,0(89,0-103,5)$ & \multirow{5}{*}{$0,010^{*}$} \\
\hline Homens & $103,5(95,0-105,0)$ & & $98,5(94,0-102,5)$ & & $96,5(95,0-104,0)$ & \\
\hline GND & & & & & & \\
\hline Mulheres & $94,0(87,0-102,0)$ & & $92,2(86,0-100,0)$ & & $93,0(85,0-99,6)$ & \\
\hline Homens & $98,0(90,5-108,0)$ & & $97,0(90,0-103,0)$ & & $98,0(87,4-104,0)$ & \\
\hline \multicolumn{7}{|c|}{$\operatorname{IMC}(\mathrm{Kg} / \mathrm{m} 2)$} \\
\hline GD & $29,7(26,6-34,2)$ & \multirow{2}{*}{$<0,000^{*}$} & $28,6(26,1-33,0)$ & \multirow{2}{*}{$0,006^{*}$} & $28,2(26,2-31,4)$ & \multirow{2}{*}{0,142} \\
\hline GND & $27,8(24,8-31,1)$ & & $27,5(24,8-30,9)$ & & $27,4(24,7-30,6)$ & \\
\hline
\end{tabular}

$\mathrm{n}$ = número de usuários; $\mathrm{GD}=$ diabéticos; $\mathrm{GND}=$ não diabéticos; $\mathrm{PAS}=$ pressão arterial sistólica; $\mathrm{PAD}=$ pressão arterial diastólica; $\mathrm{CA}$ = circunferência abdominal; IMC = índice de massa corporal; t0 = avaliação no momento de entrada; 1 = avaliação após 6 meses; t2 = avaliação após 12 meses; *nível de significância p < 0,05. 
também não apresentaram diferença estatística, mas ocorreu redução estatística da CA $(p=0,005)$, como demonstrado na Tabela 5. Os valores de PAS e PAD não mostram diferenças significativas após 12 meses ( $\mathrm{p}=0,340$ e $\mathrm{p}=0,738$, respectivamente).

\section{Discussão}

$\mathrm{Na}$ presente pesquisa, GD e GND apresentaram aderência semelhante às aulas no PAC da Regional Nordeste de Belo Horizonte, havendo relevância significativa para $\mathrm{CA}$, após 12 meses.

No mundo, por ano, a inatividade física é responsá-

Tabela 3 - Comparação da média de frequência às aulas de GD e GND no PAC, da região Nordeste de Belo Horizonte, em 2015 ( $\mathrm{n}=812$ ).

\begin{tabular}{lc}
\hline Média de frequência às aulas & $\%$ \\
\hline GD $(\mathrm{n}=114)$ & 56,7 \\
Média de frequência & \\
GND $(\mathrm{n}=698)$ & 56,2 \\
Média de frequência & \\
\hline
\end{tabular}

$\mathrm{n}$ = número de usuários; $\mathrm{GD}$ = diabéticos; $\mathrm{GND}=$ não diabéticos . vel por 5,3 milhões de mortes e por 7\% dos diagnósticos de DM2 ${ }^{15}$. No Brasil, desde 2010, a atividade física tem feito parte das ações de promoção de saúde dos municípios, através da Política Nacional de Promoção de Saúde (PNPS) ${ }^{16}$. A Secretaria Municipal de Saúde de Belo Horizonte conduziu a implementação do PAC em 2006, com a construção da primeira Academia na região leste do município. Em 2015, 60 polos estavam distribuídos nas nove regionais da cidade ${ }^{9} \mathrm{e}$, atualmente, Belo Horizonte conta com 152 centros de saúde e 78 Academias da Cidade, com aproximadamente 19.000 usuários inscritos ${ }^{17}$.

A prática regular de atividade física melhora o controle glicêmico, reduz complicações microvasculares e o risco de doenças cardiovasculares, além de contribuir para o bem-estar psicológico e a autoestima de pessoas com DM ${ }^{1,4,6,7}$. Porém, apesar da atividade física ser um importante fator na redução de morbidades, adultos com DM realizam menos exercícios do que aqueles que não possuem tal diagnóstico ${ }^{1,6,18}$.

Na presente pesquisa, GD e GND apresentaram se-

Tabela 4 - Comparação das variáveis antropométricas de usuários do GD no momento de entrada, 6 e 12 meses de inserção no PAC da região Nordeste de Belo Horizonte, em 2015 ( $\mathrm{n}=114$ ).

\begin{tabular}{|c|c|c|c|c|c|c|c|c|c|c|}
\hline \multicolumn{11}{|c|}{ Tempo } \\
\hline \multirow{3}{*}{ Variáveis } & \multicolumn{3}{|c|}{ Entrada $(\mathrm{t} 0)$} & \multicolumn{3}{|c|}{6 meses $(\mathrm{t} 1)$} & \multicolumn{3}{|c|}{12 meses $(\mathrm{t} 2)$} & \multirow{3}{*}{$\mathrm{p}$} \\
\hline & \multicolumn{3}{|c|}{$(\mathrm{n}=114)$} & \multicolumn{3}{|c|}{$(\mathrm{n}=71)$} & \multicolumn{3}{|c|}{$(n=44)$} & \\
\hline & Média & DP & Mediana & Média & $\mathrm{DP}$ & Mediana & Média & $\mathrm{DP}$ & Mediana & \\
\hline PAS (mmHg) & 129,90 & 16,42 & 130,00 & 129,11 & 15,83 & 130,00 & 130,96 & 16,47 & 130,00 & 0,836 \\
\hline $\mathrm{PAD}(\mathrm{mmHg})$ & 78,30 & 10,04 & 80,00 & 79,06 & 9,06 & 80,00 & 78,93 & 10,32 & 80,00 & 0,530 \\
\hline PESO (kg) & 74,99 & 16,20 & 72,60 & 71,73 & 14,15 & 69,75 & 69,82 & 10,60 & 70,20 & 0,466 \\
\hline CIRC AB $(\mathrm{cm})$ & 101,25 & 12,28 & 102,00 & 99,04 & 11,78 & 99,75 & 96,85 & 9,19 & 98,00 & $0,037^{*}$ \\
\hline IMC $\left(\mathrm{Kg} / \mathrm{m}^{2}\right)$ & 30,41 & 5,49 & 29,73 & 29,70 & 5,28 & 28,56 & 28,74 & 4,06 & 28,25 & 0,168 \\
\hline
\end{tabular}

$\mathrm{n}=$ número de usuários avaliados; $\mathrm{PAS}=$ pressão arterial sistólica; $\mathrm{PAD}=$ pressão arterial diastólica; $\mathrm{PESO}=$ peso corporal; $\mathrm{CIRC} \mathrm{AB}=$ circunferência abdominal; $\mathrm{IMC}=$ índice de massa corporal; $\mathrm{DP}=$ desvio padrão; $\mathrm{t} 0$ = avaliação no momento de entrada; $\mathrm{t} 1=$ avaliação após 6 meses; t2 = avaliação após 12 meses; *nível de significância $\mathrm{p}<0,05$.

Tabela 5 - Comparação das variáveis antropométricas de usuários do GND no momento de entrada, 6 e 12 meses de inserção, no PAC da região Nordeste de Belo Horizonte, em 2015 (n = 698).

\begin{tabular}{|c|c|c|c|c|c|c|c|c|c|c|}
\hline \multicolumn{11}{|c|}{ Tempo } \\
\hline \multirow{3}{*}{ Variáveis } & \multicolumn{3}{|c|}{ Entrada $(\mathrm{t} 0)$} & \multicolumn{3}{|c|}{6 meses $(t 1)$} & \multicolumn{3}{|c|}{12 meses (t2) } & \multirow{3}{*}{$\mathrm{p}$} \\
\hline & \multicolumn{3}{|c|}{$(\mathrm{n}=698)$} & \multicolumn{3}{|c|}{$(n=453)$} & \multicolumn{3}{|c|}{$(\mathrm{n}=324)$} & \\
\hline & Média & $\overline{D P}$ & Mediana & Média & $\mathrm{DP}$ & Mediana & Média & $\mathrm{DP}$ & Mediana & \\
\hline PAS (mmHg) & 125,02 & 18,23 & 121,00 & 124,39 & 16,68 & 122,00 & 124,44 & 16,63 & 122,00 & 0,340 \\
\hline $\mathrm{PAD}(\mathrm{mmHg})$ & 78,88 & 10,73 & 80,00 & 78,71 & 9,59 & 80,00 & 78,39 & 9,35 & 80,00 & 0,738 \\
\hline PESO (kg) & 69,88 & 14,38 & 68,20 & 68,79 & 13,30 & 67,30 & 67,69 & 12,58 & 66,70 & 0,877 \\
\hline CIRC AB $(\mathrm{cm})$ & 95,48 & 12,00 & 94,50 & 93,84 & 11,22 & 93,00 & 92,82 & 10,23 & 93,00 & $0,005^{*}$ \\
\hline $\operatorname{IMC}\left(\mathrm{Kg} / \mathrm{m}^{2}\right)$ & 28,19 & 5,15 & 27,76 & 27,98 & 4,83 & 27,47 & 27,71 & 4,55 & 27,40 & 0,591 \\
\hline
\end{tabular}

$\mathrm{n}=$ número de usuários avaliados; $\mathrm{PAS}=$ pressão arterial sistólica $\mathrm{PAD}=$ pressão arterial diastólica $; \mathrm{PESO}=$ peso corporal; $\mathrm{CA}=$ circunferência abdominal; $\mathrm{IMC}=$ índice de massa corporal; $\mathrm{DP}=$ desvio padrão; $\mathrm{t} 0$ = avaliação no momento de entrada; $\mathrm{t} 1$ = avaliação após 6 meses; t2 = avaliação após 12 meses; *nível de significância, p < 0,05. 
melhança em relação à média de participação às aulas, no ano de 2015 , sendo $56,7 \%$ e $56,2 \%$, respectivamente. Costa et al. ${ }^{19}$, em 2009, verificaram que a média de permanência de pessoas com DM, HAS e Obesidade em um programa de atividade física foi de 27 meses ou 43,5\% do tempo total de duração das atividades, em uma Unidade Básica de Saúde (UBS). Indivíduos que aderem às recomendações de prática de atividade física no tratamento do DM reduzem riscos de acidente vascular cerebral, insuficiência renal, amputação de membro inferior e óbito ${ }^{20}$. Ao verificar atividades de autocuidado de pessoas com DM2, Eid et al. ${ }^{21}$ identificaram o predomínio do sedentarismo nesta população, porém, também o controle metabólico e a diminuição do risco cardiovascular para aqueles que apresentavam comportamento satisfatório na prática de exercício físico.

Faria et al. ${ }^{22}$, ao avaliarem 1406 usuários da Atenção Primária à Saúde, encontraram maior adesão ao tratamento medicamentoso, alimentar e da prática de exercícios físicos para pessoas com DM que possuíam mais de 10 anos de diagnóstico da doença. Nesta pesquisa, a Planilha de Avaliação Física do PAC não apresentava o tempo de diagnóstico dos participantes com DM, limitando maiores discussões.

A questão da motivação e aderência ao exercício é multidimensional, sofrendo a influência de muitos fatores, como: experiências anteriores, apoio de familiares, aconselhamento médico, aspectos biológicos, gênero, disponibilidade de tempo, condição socioeconômica, acesso a instalações e espaços adequados, dentre outros ${ }^{23}$. Para o VIGITEL, a inatividade física está presente em $44,1 \%$ da população adulta e em $69,2 \%$ dos idosos com mais de 65 anos $^{3}$.

A melhora no metabolismo da glicose pelo exercício físico pode ocorrer, principalmente, através de três diferentes mecanismos: estimulação do transporte de glicose para o músculo; aumento na ação da insulina nas células dos órgãos envolvidos no exercício; regulação positiva da via de sinalização pela insulina, como resultado de exercício físico regular ${ }^{1,4-7}$. A combinação exercícios físicos aeróbicos e de resistência é mais eficaz na melhoria do controle glicêmico, na ação da insulina e na oxidação de gorduras ${ }^{1,6,7}$. Os exercícios aeróbicos, de intensidade moderada, também apresentam benefícios na circunferência da cintura, na glicose sanguínea e PAD de pessoas com DM, quando praticados em pelo menos em três dias da semana ${ }^{24}$. Em 2015, a prescrição de exercícios no PAC, em Belo Horizonte, intercalava dias de exercícios combinados com dias de exercícios aeróbicos.
Uma característica comum no DM2 é o excesso de gordura corporal ${ }^{1,6}$. Nesta pesquisa, ao compararmos as variáveis antropométricas de GD e GND, verifica-se que, no momento de entrada, os valores de P, CA e IMC de GD foram significativamente maiores que GND. Porém, ao final de 12 meses de participação no PAC, a diferença significativa entre os grupos não prevaleceu para $\mathrm{P}$ e IMC. Avaliando-se dados de UBS em Presidente Prudente (SP), após 18 meses, Araujo et al. ${ }^{25}$ concluíram que os participantes com DM2 e participantes que desenvolveram DM2 durante o acompanhamento, mostraram maiores valores de IMC, circunferência da cintura, gastos com saúde e foram menos ativos quando comparados aos participantes sem diagnóstico de DM2.

Nesta pesquisa, no momento de entrada, a mediana de IMC de GD correspondeu a 29,73 Kg/m² e, ao final do ano, tal valor foi $28,25 \mathrm{Kg} / \mathrm{m}^{2}$. Assim, apesar da redução, indivíduos com DM prevaleceram com o perfil de sobrepeso. O PAC em Belo Horizonte, por semana, oferta três aulas de exercícios físicos, com duração de 60 minutos cada (180 minutos/semana). Para o American College of Sports Medicine (ACSM), a maioria dos indivíduos com DM2 estão acima do peso, o que leva o Profissional de Educação Física a também adotar diretrizes de sobrepeso e obesidade na prescrição do exercício físico. Assim, para manter a perda de peso a longo prazo, indivíduos com DM2 e sobrepeso ou obesidade devem aumentar a quantidade de exercícios de intensidade moderada a vigorosa para, pelo menos, 250 minutos/semana ${ }^{6}$.

Diante a média de presença às aulas de 2015, GD obteve redução de $\mathrm{P}$ e IMC, além de diminuição significativa de $\mathrm{CA}$, após 12 meses de participação.

Para adultos com DM2 e sobrepeso ou obesidade, a perda de peso modesta ( $5 \%$ do peso inicial) tem demonstrado bons resultados no controle glicêmico e na redução de medicamentos antidiabéticos, sendo que dieta e atividade física devem ser programadas para atingir uma perda de 5 a $10 \%$ do peso inicial ${ }^{1,6}$. Nesta pesquisa, ao compararmos a mediana de $\mathrm{P}$, no momento de entrada e após 12 meses, indivíduos de GD reduziram 3,3\% do peso inicial.

Com o objetivo de analisar os efeitos de oito anos de participação regular em um programa de exercícios sobre a pressão arterial e a mortalidade no sistema brasileiro de saúde pública, Turi et al. ${ }^{26}$ identificaram uma mortalidade $29,4 \%$ menor para os participantes ativos que apresentavam HAS e/ou DM, assim como, uma menor 
quantidade de consultas médicas e valores de PAD.

O controle da HAS representa meta prioritária para redução de risco cardiovascular e renal em indivíduos com DM, sendo 130 × $80 \mathrm{mmHg}$ o limite pressórico para aqueles com diagnóstico de $\mathrm{DM}^{1}$. Nesta pesquisa, no momento de entrada, os valores de PAS e PAD foram 130 e $80 \mathrm{mmHg}$, respectivamente, mantendo-se ao longo dos 12 meses de acompanhamento no PAC. Diretrizes para o gerenciamento da HAS também enfatizam a cessação do tabagismo, a redução da ingestão de sódio, moderação do consumo de álcool e hábitos alimentares saudáveis ${ }^{6}$.

Intervenções nutricionais aliadas à atividade física resultou em melhor adequação no consumo de embutidos e mudanças positivas na antropometria, em usuários do PAC, de Belo Horizonte. Neste estudo de Machado et $\mathrm{al}^{27}$, os atendimentos nutricionais foram realizados por acadêmicos de Nutrição, com duração de, aproximadamente, uma hora e ao longo de seis meses. No PAC, em Belo Horizonte, as ações de promoção em saúde não possuem uma metodologia definida, ao contrário do que ocorre com a prescrição dos exercícios físicos. Assim, orientações nutricionais podem ocorrer de formas e frequências distintas, em diferentes Academias, a depender do Profissional de Educação Física responsável pelo espaço e das características do território.

O controle da obesidade e intervenções em HAS dislipidemia e sedentarismo previnem o aparecimento de DM2 e doenças cardiovasculares ${ }^{1}$. $\mathrm{Na}$ cidade de Bauru (SP), a prática de caminhada foi a atividade física mais referida por usuários do SUS. Além disso, grupos de maior prática de caminhada apresentaram menores valores para idade, IMC, circunferência da cintura e gastos com medicamentos ${ }^{28}$. Apesar do registro do uso de medicamento estar incluso na Planilha de Avaliação Física do PAC, em Belo Horizonte, esse apresentava ausência de detalhes quanto a nome, dosagem ou a hora do dia que a ingestão era recomendada, dificultando análises mais profundas.

Nesta pesquisa, considerando os valores da mediana, GND apresenta redução de P, CA e IMC, com relevância significativa para $\mathrm{CA}$. Além disso, em relação ao $P$ inicial, indivíduos de GND apresentaram redução de 2,19\%. Valores de PAS aumentaram após 12 meses de participação no PAC, sem relevância significativa.

Um programa de condicionamento físico supervisionado por uma equipe multiprofissional para normotensos e pré-hipertensos, após 24 meses, promoveu a redução da PAS e PAD, acompanhados da redução da circunferência da cintura, representando uma alternativa eficaz na prevenção a patologias relacionadas ao excesso de adiposidade ${ }^{29}$. Intervenções comunitárias que promovem a mudança no estilo de vida das pessoas possuem um custo benefício interessante e são tão eficazes quanto outras práticas para reduzir a possibilidade de futuros diagnósticos em $\mathrm{DM}^{30}$.

Apesar de haver evidências que adultos com DM realizam menos exercícios do que aqueles que não possuem tal diagnóstico ${ }^{1,6}$, nesta pesquisa, GD e GND apresentaram aderência semelhante às aulas no $\mathrm{PAC}$ da Regional Nordeste de Belo Horizonte, com redução de P, CA e IMC, havendo relevância significativa para CA, após 12 meses. Além disso, diferenças antropométricas significativas encontradas entre GD e GND, na entrada ao PAC, não prevaleceram para $\mathrm{P}$ e IMC, ao final do ano analisado.

Talvez o estudo de um número maior de usuários, ou por um tempo maior, ou de maior aderência, possa trazer resultados mais robustos. Além disso, como o GD apresentou mediana de IMC compatível com sobrepeso, um volume maior de exercícios físicos ou maior presença às aulas do PAC, como orientado pelo ACSM, fossem necessários para melhor impacto nos resultados. Sugere-se a inclusão do tempo de diagnóstico de DM dentre as informações coletadas pela Planilha de Avaliação Física do PAC, em Belo Horizonte, bem como um registro mais adequado dos medicamentos utilizados pelos participantes. Por fim, usuários do Programa poderiam apresentar maiores reduções antropométricas se também houvesse uma metodologia única de orientações para uma alimentação saudável.

\section{Conflito de interesse}

Os autores declaram não haver conflito de interesse.

\section{Contribuição dos autores}

Jubilini DC, foi responsável pela concepção inicial do estudo, coleta de dados, busca na literatura e redação e revisão crítica do texto. Bosco AP, foi responsável pela concepção inicial do estudo, redação e revisão crítica do texto.

\section{Agradecimentos}

Os autores agradecem a Secretaria Municipal de Saúde de Belo Horizonte por viabilizar o acesso às planilhas de avaliação física e às chamadas do Programa Academia da Cidade.

\section{Referências}

1. Sociedade Brasileira de Diabetes. Diretrizes da Sociedade Brasileira de Diabetes: 2017-2018. São Paulo: Editora Clannad; 2017. 
2. International Diabetes Federation. IDF Atlas. 7th ed. Brussels, Belgium: International Diabetes Federation; 2015.

3. Brasil. Ministério da Saúde. Secretaria de Vigilância em Saúde. Departamento de Análise em Saúde e Vigilância de Doenças não Transmissíveis. Vigitel Brasil 2018. Brasília: Ministério da Saúde; 2019.

4. Coelho CF, Burini RC. Atividade física para prevenção e tratamento das doenças crônicas não transmissíveis e da incapacidade funcional. Rev Nutr. 2009;22(6):937-46.

5. Matsudo SM, Matsudo VR, Neto TLB. Efeitos benéficos da atividade física na aptidão física e saúde mental durante o processo de envelhecimento. Rev Bras Ativ Fís Saúde. 2000;5(2):60-6

6. Colberg SR, Sigal RJ, Fernhall B, Regensteiner JG, Blissmer BJ, Rubin RR, et al. Exercise and Type 2 Diabetes - The American College of Sports Medicine and the American Diabetes Association: joint position statement. Diabetes Care. 2010;33:2692-6.

7. Lemos ET, Nunes S, Teixeira, F, Reis, F. Regular physical exercise training assists in preventing type 2 diabetes development: focus on its antioxidant and anti-inflammatory properties. Cardiovasc diabetol. 2011;10:12.

8. Forechi L, Mill JG, Griep RH, Santos I, Pitanga F, Molina MCB. Adherence to physical activity in adultsvwith chronic diseases: ELSA-Brasil. Rev. Saúde Públ. 2018;52:31.

9. Fernandes AP, Andrade ACS, Ramos CGC, Friche AAL, Dias MAS, Xavier CC, et al. Atividade física de lazer no território das Academias da Cidade, Belo Horizonte, Minas Gerais, Brasil: o efeito da presença de um programa de promoção da saúde na comunidade. Cad. Saúde Pública. 2015;31:1-13.

10. Brasil. Ministério da Saúde. Secretaria de Vigilância em Saúde. Avaliação de Efetividade de Programas de Atividade Física no Brasil. Brasília; 2011.

11. Liz CM, Crocetta TB, Viana MS, Brandt R, Andrade A. Aderência à prática de exercícios físicos em academias de ginástica. Motriz. 2009;16(1):181-8.

12. Mendes TAB, Goldbaum M, Segri NJ, Barros MBA, Cesar CLG, Carandina L, et al. Diabetes mellitus: fatores associados à prevalência em idosos, medidas e práticas de controle e uso dos serviços de saúde em São Paulo, Brasil. Cad. Saúde Pública. 2011;27(6):1233-43.

13. Silva KL, Sena R, Matos J,Lima K, Silva P. Acesso e utilização da Academia da Cidade de Belo Horizonte: perspectiva de usuários e monitores. Rev Bras Ativ Fís Saúde. 2014;19 (6):700-10.

14. Costa, BVL, Mendonça RD, Santos LC, Peixoto SV, Alves M, Lopes ACS. Academia da Cidade: um serviço de promoção da saúde na rede assistencial do Sistema Único de Saúde. Ciênc. Saúde Colet. 2013;18(1):95-102.

15. Lee I-Min, Shiroma EJ, Lobelo F, Puska P, Blair SN, Katzmarzyk PT. Impact of physical inactivity on the world's major non-communicable diseases. Lancet. 2012;380(9838): 219-29.

16. Brasil. Ministério da Saúde. Secretaria de Vigilância em Saúde. Secretaria de Atenção à Saúde. Política Nacional de Promoção da Saúde. Brasília: Ministério da Saúde; 2010
17. Anais eletrônicos. Belo Horizonte: Prefeitura de Belo Horizonte; 2020. [citado em 2020 mai 27]. Disponível em: https://prefeitura.pbh.gov.br/saude.

18. Thomas N, Alder E, Leese GP. Barriers to physical activity in patients with diabetes. Postgrad Med J. 2004;80:287-91.

19. Costa BV, Bottcher LB, Kokubun E. Adesão a um programa de atividade física e fatores associados. Motriz. 2009;15(1):25-36.

20. Chen Y, Sloan FA, Yashkin AP. Adherence to diabetes guidelines for screening, physical activity and medication and onset of complications and death. J Diabetes Complications. 2015; 29(8):1228-33.

21. Eid LP, Leopoldino SAD, Oller GASAO, Pompeo DA, Martins MA, Gueroni LPB. Fatores relacionados às atividades de autocuidado de pacientes com diabetes mellitus tipo 2. Esc Anna Nery. 2018;22(4):e20180046.

22. Faria HTG, Rodrigues FFL, Zanetti ML, Araújo MFM, Damasceno MMC. Fatores associados à adesão ao tratamento de pacientes com diabetes mellitus. Acta Paul Enferm. 2013; 26(3):231-7

23. Assunção TS, Ursine PGS. Estudo de fatores associados à adesão ao tratamento não farmacológico em portadores de diabetes mellitus assistidos pelo Programa Saúde da Família, Ventosa, Belo Horizonte. Ciênc. Saúde Colet. 2008;13:2189-97.

24. De Sá CA, Heizenb PG, Corraloa VS, Santos GAG, Soares NMM. Chronic effect of aerobic exercise on anthropometric, biochemical and hemodynamic variables in individuals with type 2 diabetes mellitus: a systematic review. Rev Andal Med Deporte. 2016;9(4):173-79.

25. Araujo MYC, Turi BC, Queiroz DC, Ferro IS, Bortolatto CR, Codogno JS. Type 2 diabetes, healthcare expenditures and its correlation with anthropometric factors and physica activity: 18-month follow-up in a Brazilian city. Motriz. 2018;24(1):e101867.

26. Turi BC, Bonfim MR, Codogno JS, Fernandes RA, Araújo LGM, Amaral SL, et al. Exercise, blood pressure and mortality: findings of eight years of follow-up. Rev Bras Med Esporte. 2017;23(2):133-6.

27. Machado CH, Carmo AS, Horta PM, Lopes ACS, Santos LC. Efetividade de uma intervenção nutricional associada à prática de atividade física. Cad. Saúde Colet. 2013;21(2): 148-53.

28. Turi BC, Codogno JS, Fernandes RA, Monteiro HL. Caminhada e gastos com saúde em adultos usuários do sistema público de saúde brasileiro: estudo transversal retrospectivo. Ciênc. Saúde Colet. 2015;20(11):3561-8.

29. Zaar A, Reis VM, Sbardelotto ML. Efeitos de um programa de exercícios físicos sobre a pressão arterial e medidas antropométricas. Rev Bras Med Esporte. 2014;20(1):13-6.

30. Jacobs-Van Der B, Bos G, Bemelmans WJ, Hoogenveen RT, Vijgen SM, Baan CA. Lifestyle Interventions Are CostEffective in People With Different Levels of Diabetes Risk. Diabetes Care. 2007;30(1):128-34.

Recebido: 26/07/2020

Aprovado: 24/09/2020

Como citar este artigo:

Jubilini DC, Bosco A. Impacto e aderência de individuos com diabetes no Programa Academia da Cidade. Rev Bras Ativ Fís Saúde. 2020;25:e0136. DOI: 10.12820/rbafs.25e0136 